\title{
Can Gut Microbiota Modulation Be Used as a Practical Treatment for Obesity?
}

\author{
Jeong Ki Paek, Seon Yeong Lee* \\ Department of Family Medicine, Sanggye Paik Hospital, Inje University College of Medicine, Seoul, Korea
}

It is well known that interactions between behavioral factors, specifically, energy intake and physical activity, environmental, and genetic factors can impact the development of obesity. Recently, the composition of gut microbiota has emerged as a possible important endogenous factor that influences nutrient acquisition and energy regulation. Knowledge and understanding about the association between the human gut microbiota and obesity has increased exponentially during last decade, and the concept of gut microbiota modulation has provided a new paradigm for obesity treatment. Mutual and complex mechanisms are involved in the relationship between the gut microbiota, environment and obesity, but these mechanisms still remain largely unexplored. Understanding the mechanisms for how gut microbiota modulate host appetite and metabolism can provide clues for new strategies for obesity treatment. In this context, much effort has been made to manipulate gut microbiota to target host metabolism and appetite control.

Administration of live beneficial bacterial strains (probiotics) is a relatively safe and noninvasive approach for altering the gut microbiota in humans. Several studies have reported that particular bacterial strains could reduce body weight or metabolic disor- ders, such as metabolic endotoxemia and insulin resistance. Zhang et al. ${ }^{1}$ indicated that probiotic consumption could reduce weight and body mass index (BMI) when multiple species of probiotics were administrated. However, there is currently no evidence of compositional alterations of the fecal microbiota in response to probiotic administration. ${ }^{2}$ Recently, Borgeraas et al. ${ }^{3}$ reported a meta-analysis of randomized controlled trials, which were conducted to examine the effects of probiotic administration in only overweight (BMI, $\left.25-29.9 \mathrm{~kg} / \mathrm{m}^{2}\right)$ or obese (BMI, $\left.\geq 30 \mathrm{~kg} / \mathrm{m}^{2}\right)$ adults. The study showed that short-term $(\leq 12$ weeks) probiotic supplementation reduced body weight: weighted mean difference ( $95 \%$ confidence interval) of body weight is $-0.60 \mathrm{~kg}\left(-1.19\right.$ to $\left.-0.01 \mathrm{~kg}, \mathrm{I}^{2}=49 \%\right) ; \mathrm{BMI},-0.27 \mathrm{~kg} / \mathrm{m}^{2}(-0.45$ to $\left.-0.08 \mathrm{~kg} / \mathrm{m}^{2}, \mathrm{I}^{2}=57 \%\right)$; and fat percentage, $-0.60 \%(-1.20 \%$ to $\left.-0.01 \%, \mathrm{I}^{2}=19 \%\right)$. Prebiotics are non-indigestible fibers, which undergo bacterial fermentation and subsequently stimulate the growth of particular types of bacteria, thus conferring a beneficial effect on the host. Parnell and Reimer ${ }^{4}$ suggested that oligofructose supplementation over 12 weeks reduced body weight, mainly body fat, in overweight and obese subjects. The mechanism for this effect is hypothesized to be associated with in- 
creased short chain fatty acids (SCFAs) and anorexigenic peptides, such as, peptide YY (PYY) and glucagon-like peptide-1 (GLP-1) production.

Over the past decades, pervasive use of antibiotics has been found to disrupt the microbiota composition. The increased use of antibiotics has also been paralleled with an increase in the prevalence of obesity. Several studies have recently shown that repeated exposure to antibiotics in early life is associated with early childhood obesity. ${ }^{5}$ In comparison, antibiotic use has been shown to improve plasma lipopolysaccharides (LPS) levels and metabolic endotoxemia in animal studies. ${ }^{6}$ The mechanisms for microbiota effects on host body composition induced by antibiotic treatment are complex and remain ambiguous. However, if antibiotic administration can be optimized, this approach could provide a tool to modulate gut microbiota and improve metabolic disorders and obesity.

A fecal microbiota transplant (FMT) is the process transplanting fecal bacteria from a healthy individual into a recipient to cure a specific disease, which is an efficient intervention to alter the gut microbial ecosystem. FMT is a treatment strategy for inflammatory bowel disease, irritable bowel syndrome and metabolic diseases, in addition to severe recurrent Clostridium difficile infection. Recently, Kootte et al. 7 reported that there were beneficial effects of lean donor FMT on glucose metabolism, which is dependent on decreased fecal microbial diversity at baseline. Therefore, FMT could prove to be a useful treatment against obesity and metabolic disorder in the near future although the procedure is not well-established and the mechanisms require further research investigation.

Several mechanisms for gut microbiota modulation, which could potentially influence weight gain and fat deposition, have been elucidated in many studies. Altered symbiotic interactions between the gut microbiota and the host are associated with increased metabolic and immune disorders. Turnbaugh et al. ${ }^{8}$ suggested an "energy harvest" hypothesis which proposes that the obese microbiome (microbial metagenome sequences) have an increased capacity to harvest energy from the diet. Gut microbiota produce SCFAs from remnant dietary fibers, subsequently modulating the microbiome-gut-brain axis. SCFAs (acetate, propionate, and butyrate) are ligands for G-protein-coupled recep- tors (GPCRs), which are regulators of gut motility, host energy balance, fat storage and appetite. 9 SCFAs affect appetite through anorexigenic peptides (PYY, GLP-1). ${ }^{10} \mathrm{PYY}$ is stimulated by GPCRs which can also lead to changes in gut motility and facilitation of nutrient absorption. Acetate in particular can activate the parasympathetic nervous system that increases glucose-stimulated insulin, ghrelin secretion, and excessive eating. ${ }^{11}$ Butyrate may reduce the appetite by regulating leptin expression in adipocytes. $^{12}$

In addition, gut microbiota affect the expression of obesity-related genes, for example, fasting-induced adipose factor (FIAF) and AMP-activated protein kinase (AMPK). ${ }^{13,14}$ Overexpression of FIAF can reduce adipose tissue by stimulating fatty acid oxidation and uncoupling in fat. AMPK inhibition can lead to decreased fatty acid oxidation and upregulation of lipogenesis. Chronic low-grade inflammation is prominent in both obesity and insulin resistance. A high-fat diet can increase gut permeability and the proportion of LPS-containing microbiota in the gut. ${ }^{15}$ Excessively high levels of LPS (metabolic endotoxemia) can lead to gut, hepatic, and adipose tissue inflammation and diabetes.

Although the rapidly evolving metagenomics field has facilitated a better understanding of microbiome-host-environment interaction, only a fraction of the complex and dynamic relationships have been revealed. Some of the mechanisms for gut microbiota and obesity have already been identified, including energy harvest, free fatty acid modulation, obesity-related gene expression and metabolic endotoxemia. In accordance with those theories, modifications in the gut microbial ecology by probiotics, prebiotics, antibiotics or FMT can change and possibly improve host obesity and metabolic diseases. Therefore, integrating epidemiological analyses in practice will enable researchers to optimize therapeutic strategies to modulate gut microbiota composition to target metabolic effects as well as obesity. In the foreseeable future, we hope to develop additional tailored therapeutic strategies for obesity and metabolic disorders based on individual gut microbiota compositions, host, and environmental factors.

\section{CONFLICTS OF INTEREST}

The authors declare no conflict of interest. 


\section{REFERENCES}

1. Zhang Q, Wu Y, Fei X. Effect of probiotics on body weight and body-mass index: a systematic review and meta-analysis of randomized, controlled trials. Int J Food Sci Nutr 2015;67: 571-80.

2. Kristensen NB, Bryrup T, Allin KH, Nielsen T, Hansen TH, Pedersen O. Alterations in fecal microbiota composition by probiotic supplementation in healthy adults: a systematic review of randomized controlled trials. Genome Med 2016;8:52.

3. Borgeraas H, Johnson LK, Skattebu J, Hertel JK, Hjelmesaeth $\mathrm{J}$. Effects of probiotics on body weight, body mass index, fat mass and fat percentage in subjects with overweight or obesity: a systematic review and meta-analysis of randomized controlled trials. Obes Rev 2018;19:219-32.

4. Parnell JA, Reimer RA. Weight loss during oligofructose supplementation is associated with decreased ghrelin and increased peptide YY in overweight and obese adults. Am J Clin Nutr 2009;89:1751-9.

5. Bailey LC, Forrest CB, Zhang P, Richards TM, Livshits A, DeRusso PA. Association of antibiotics in infancy with early childhood obesity. JAMA Pediatr 2014;168:1063-9.

6. Cani PD, Bibiloni R, Knauf C, Waget A, Neyrinck AM, Delzenne NM, et al. Changes in gut microbiota control metabolic endotoxemia-induced inflammation in high-fat diet-induced obesity and diabetes in mice. Diabetes 2008;57:1470-81.

7. Kootte RS, Levin E, Salojärvi J, Smits LP, Hartstra AV, Udayappan SD, et al. Improvement of insulin sensitivity after lean donor feces in metabolic syndrome is driven by baseline intestinal microbiota composition. Cell Metab 2017;26:611-9.e6.

8. Turnbaugh PJ, Ley RE, Mahowald MA, Magrini V, Mardis
ER, Gordon JI. An obesity-associated gut microbiome with increased capacity for energy harvest. Nature 2006;444:102731.

9. Samuel BS, Shaito A, Motoike T, Rey FE, Backhed F, Manchester JK, et al. Effects of the gut microbiota on host adiposity are modulated by the short-chain fatty-acid binding $\mathrm{G}$ protein-coupled receptor, Gpr41. Proc Natl Acad Sci U S A 2008; 105:16767-72.

10. Delzenne NM, Neyrinck AM, Cani PD. Modulation of the gut microbiota by nutrients with prebiotic properties: consequences for host health in the context of obesity and metabolic syndrome. Microb Cell Fact 2011;10 Suppl 1:S10.

11. Perry RJ, Peng L, Barry NA, Cline GW, Zhang D, Cardone $\mathrm{RL}$, et al. Acetate mediates a microbiome-brain- $\beta$-cell axis to promote metabolic syndrome. Nature 2016;534:213-7.

12. Soliman MM, Ahmed MM, Salah-Eldin AE, Abdel-Aal AA. Butyrate regulates leptin expression through different signaling pathways in adipocytes. J Vet Sci 2011;12:319-23.

13. Mandard S, Zandbergen F, van Straten E, Wahli W, Kuipers F, Müller M, et al. The fasting-induced adipose factor/angiopoietin-like protein 4 is physically associated with lipoproteins and governs plasma lipid levels and adiposity. J Biol Chem 2006;281:934-44.

14. Bäckhed F, Manchester JK, Semenkovich CF, Gordon JI. Mechanisms underlying the resistance to diet-induced obesity in germ-free mice. Proc Natl Acad Sci U S A 2007;104:97984.

15. Cani PD, Amar J, Iglesias MA, Poggi M, Knauf C, Bastelica D, et al. Metabolic endotoxemia initiates obesity and insulin resistance. Diabetes 2007;56:1761-72. 